\title{
ARTICLE
}

Acute myeloid leukemia

\section{Inhibition of ubiquitin-specific protease 7 sensitizes acute myeloid leukemia to chemotherapy}

\author{
Maëlle Cartel ${ }^{1,2} \cdot$ Pierre-Luc Mouchel ${ }^{1,3,4} \cdot$ Mathilde Gotanègre ${ }^{1,3} \cdot$ Laure David $^{1,2,7} \cdot$ Sarah Bertoli ${ }^{1,2,4}$. \\ Véronique Mansat-De Mas ${ }^{1,2,5} \cdot$ Arnaud Besson $\mathbb{1}^{6} \cdot$ Jean-Emmanuel Sarry ${ }^{1,3} \cdot$ Stéphane Manenti ${ }^{1,2}$. \\ Christine Didier $\mathbb{( D D}^{1,2}$
}

Received: 11 October 2019 / Revised: 13 May 2020 / Accepted: 13 May 2020 / Published online: 23 May 2020

(c) The Author(s), under exclusive licence to Springer Nature Limited 2020

\begin{abstract}
Resistance of acute myeloid leukemia (AML) to therapeutic agents is frequent. Consequently, the mechanisms leading to this resistance must be understood and addressed. In this paper, we demonstrate that inhibition of deubiquitinylase USP7 significantly reduces cell proliferation in vitro and in vivo, blocks DNA replication progression and increases cell death in AML. Transcriptomic dataset analyses reveal that a USP7 gene signature is highly enriched in cells from AML patients at relapse, as well as in residual blasts from patient-derived xenograft (PDX) models treated with clinically relevant doses of cytarabine, which indicates a relationship between USP7 expression and resistance to therapy. Accordingly, singlecell analysis of AML patient samples at relapse versus at diagnosis showed that a gene signature of the pre-existing subpopulation responsible for relapse is enriched in transcriptomes of patients with a high USP7 level. Furthermore, we found that USP7 interacts and modulates CHK1 protein levels and functions in AML. Finally, we demonstrated that USP7 inhibition acts in synergy with cytarabine to kill AML cell lines and primary cells of patients with high USP7 levels. Altogether, these data demonstrate that USP7 is both a marker of resistance to chemotherapy and a potential therapeutic target in overcoming resistance to treatment.
\end{abstract}

These authors contributed equally: Stéphane Manenti, Christine Didier

Supplementary information The online version of this article (https:// doi.org/10.1038/s41375-020-0878-x) contains supplementary material, which is available to authorized users.

Christine Didier

christine.didier@inserm.fr

1 Cancer Research Center of Toulouse, INSERM U1037, CNRS ERL 5294, Université de Toulouse, Toulouse, France

2 Ligue Nationale Contre le Cancer, équipe labellisée 2016, Toulouse, France

3 Ligue Nationale Contre le Cancer, équipe labellisée 2018, Toulouse, France

4 Service d'hématologie, Institut Universitaire du Cancer de Toulouse-Oncopôle, Toulouse, France

5 Laboratoire d'hématologie, Institut Universitaire du Cancer de Toulouse-Oncopole, Toulouse, France

6 LBCMCP, Centre de Biologie Intégrative, Université de Toulouse, CNRS, UPS, 31062 Toulouse Cedex, France

7 Present address: IRSD, Université de Toulouse, INSERM, INRA, ENVT, UPS, Toulouse, France

\section{Introduction}

Acute myeloid leukemia (AML) originates from the transformation and clonal expansion of undifferentiated hematopoietic progenitors, characterized by altered growth, differentiation, and proliferation capacities, which result in failure of bone marrow hematopoietic functions. Although a majority of AML patients initially respond to standard induction therapy, a protocol that combines cytarabine (AraC) with an anthracycline, relapses are common and overall survival remains very poor [1]. Whole-genome sequencing analyses have highlighted the molecular heterogeneity of AML and allow for risk-based stratification [2]. For some mutations resulting in oncogenic signaling, specific inhibitors have been developed and are included in clinical strategies [3, 4]. Unfortunately, in the majority of cases these strategies show only transient and limited efficacy due to a variety of resistance mechanisms that occur in minor subpopulations of resistant leukemic cells which can initiate relapse [5]. Consequently, identification of these resistance mechanisms and new potential drug targets is urgently needed in AML. 
Ubiquitylation is an essential post-translational modification involved in protein stability, localization, interactions, and activity. This process is closely regulated, antagonized by cysteine protease deubiquitinating enzymes (or DUBs), and is involved in multiple signaling [6]. Ubiquitin-specific protease 7 (USP7, also known as herpesvirus-associated ubiquitinspecific protease, or HAUSP) belongs to the largest subfamily of DUB and is one of the most studied, disease-associated DUBs [7]. It has been noted that USP7 participates in the regulation of apoptosis and senescence by modulating the p53 pathway, either by directly deubiquitinating p53 or by stabilizing MDM2, an E3-ubiquitin ligase that ubiquitinates and targets p53 for proteasomal degradation [8]. Independently of its action on p53, USP7 modulates various pathways in homeostasis or during oncogenesis by targeting a large panel of substrates involved in cell cycle, DNA damage response, immune response, signal transduction, neuronal differentiation, and epigenetic regulation [7, 9]. Other notable targets of USP7 include the tumor suppressor PTEN [10] and the transcription factor FOXO4 [11]. Through deubiquitination, USP7 triggers their nuclear exclusion and inactivation. Interestingly, in chronic myeloid leukemia, BCR-ABL phosphorylates USP7 and enhances its deubiquitinating activity on PTEN [12]. Therefore, it is evident that USP7 is at the center of a complex network, and recent efforts have focused on the discovery and development of small molecule inhibitors of this protein [13-19]. These inhibitors were found to enhance apoptosis in chronic lymphocytic leukemia [20] and multiple myeloma [21] and to reduce neuroblastoma growth in vivo [22]. In this context, we hypothesize that a possible dysregulation of USP7 may contribute to resistance in AML.

In this study, we first showed that pharmacological or RNA interference-mediated inhibition of USP7 significantly reduced AML cell proliferation in vitro and in vivo, impaired replication fork progression and increased AML cell death. Furthermore, our results demonstrate that USP7 expression is a predictive marker of resistance to chemotherapy in AML. We also report that USP7 is a major regulator of $\mathrm{CHK} 1$ protein in AML through its deubiquitinating activity, which leads to $\mathrm{CHK} 1$ stabilization. Finally, we found that USP7 inhibition potentiates cytarabine (AraC) treatment by increasing leukemic cell death, and independently of p53 status. Altogether, these results suggest that targeting USP7 may represent a novel therapeutic strategy in AML.

\section{Materials and methods}

\section{Proximity ligation assay (PLA)}

For PLA, cells were washed twice with $1 \times$ PBS, fixed with $4 \%$ PFA and then permeabilized. AML cells were seeded on coverslips pretreated with Cell Tack (ref 354240, lot 7033571, Corning). Using the Duolink kit by Sigma, the following steps are described according to Adam et al. [23]. The PLA signal was detected with a Zeiss confocal microscope (LSM 780). A series of Z-stack confocal microscopy images was taken, and quantification of the images was carried out using ImageJ software.

For immunofluorescence, the staining protocol used is described by David et al. [24].

\section{Clonogenic assay}

Clonogenic assays were performed on different AML primary samples, as previously described [24].

\section{Tumor xenografts in NOD SCID gamma (NSG) mice}

NOD/LtSz-SCID/ IL-2R $\gamma$ chain null (NSG) mice were bred at the UMS006 in Toulouse (France) using breeders obtained from Charles River. All animal experiment protocols were approved by the Institutional Animal Care and Use Ethics Committee of the UMS006 and the MidiPyrénées Region (approval 2017071314596526). Mice (6-9 weeks old) were sublethally treated with busulfan (30 $\mathrm{mg} / \mathrm{kg}$ ) $24 \mathrm{~h}$ before injection of leukemic cells. Leukemic OCI-AML3 cell line was thawed at room temperature, washed twice in PBS, and suspended in Hanks balanced salt solution at a final concentration of 2 million cells per $200 \mu \mathrm{l}$ of Hanks balanced salt solution per mouse for tail vein injection. Twenty-two days after injection, the mice were randomly split into two groups: one group was treated with $100 \mu \mathrm{l}$ corn oil with $10 \%$ DMSO as a vehicle $(n=7)$, and one group was treated with $30 \mathrm{mg} / \mathrm{kg}$ of P22077 diluted in $100 \mu$ corn oil with $10 \%$ DMSO $(n=8)$. Mice were treated by daily IP injections of P22077 or the vehicle for 5 days. Daily monitoring of mice for symptoms of disease (ruffled coat, hunched back, weakness and reduced mobility) determined the time to euthanize animals with signs of distress. NSG mice were euthanized in accordance with European ethics committee protocols. Overall mouse survival was established under these conditions.

For patient-derived xenograft (PDX), NSG mice (6-9 weeks old) were sublethally treated with busulfan $(30 \mathrm{mg} / \mathrm{kg}) 24 \mathrm{~h}$ before injection of human leukemic cells. Mice were engrafted by an injection of $3 \cdot 10^{6}$ primary AML cells in the tail vein. Twenty-two weeks after injection, peripheral blood was obtained to determine the fraction of human blasts using flow cytometry. Mice were then randomly split into two groups: one group was treated with 100 $\mu \mathrm{l}$ PBS containing $10-15 \%$ DMSO as a vehicle $(n=6)$, and one group was treated with $15 \mathrm{mg} / \mathrm{kg}$ of P22077 diluted in $100 \mu \mathrm{l}$ PBS with DMSO $(n=7)$. Mice were treated 3 days per week for 2 weeks and daily for 1 week by IP injections. 
NSG mice were euthanized in accordance with European ethics committee protocols. Bone marrow (mixed from tibias and femurs) and spleen were dissected in a sterile environment and flushed in Hanks balanced salt solution with $1 \%$ FBS. Blasts from bone marrow and spleen were labeled with FITC-conjugated anti-hCD3, PE-conjugated antihCD33, PerCP-Cy5.5-conjugated anti-mCD45.1, and APCH7-conjugated anti-hCD45 (all antibodies from Becton Dickinson, BD, except for FITC-conjugated anti-hCD3 from Ozyme Biolegend) to determine the fraction of human blasts (hCD45+mCD45.1-hCD33+ cells) using flow cytometry. Analyses were performed on a Life Science Research II (LSR II) flow cytometer with DIVA software (BD). The number of AML cells in the total tumor burden (in bone marrow and spleen) was determined by using CountBright beads (Invitrogen) and applying the described protocol.

\section{RNA sequence analysis}

\section{Single-cell transcriptomics}

A blood cell sample was collected from one patient at diagnosis and at relapse following chemotherapeutic induction. AML cells were purified by Ficoll centrifugation and AML blast cells were then sorted based on the expression of CD45+, CD33+ and annexinV-. Five hundred cells were used per condition for 10x Genomics single cell assaying as recommended by $10 \mathrm{x}$ Genomics. Sequencing was performed with an Illumina High seq 3000 . The raw data were clustered with the 10x Genomics cell ranger pipeline.

\section{Transcriptomic signatures and data mining}

Two USP7 signatures were generated from transcriptomes of AML patients with high versus low expression of USP7 from two independent databases (TCGA Network [25], and Verhaak database GSE6891 [26]). Gene Set Enrichment Analysis (GSEA) using USP7 signature was performed from the relapse and diagnosis transcriptomes according to Hackl et al. [27], the high and low responder in mice according to Farge T et al. [28], and the LSC and bulk transcriptomes according to Eppert et al. [29]. The analysis was performed using the Broad Institute GSEA3.0 software.

\section{DNA fiber spreading}

DNA fiber spreading was performed on leukemic cell line, as previously described [24].

\section{Statistical analysis}

At least three independent experiments were carried out to generate each dataset and statistical analyses were performed as described in the figures, using the Prism software package (GraphPad Software). Results are expressed as mean \pm SEM. Differences were considered significant for the following $p$ values: $* P<0.05$; $* * P<0.01$; $* * * P<0.001$; $* * * * P<0.0001$.

\section{Results}

\section{Targeting USP7 in leukemic cell lines}

We first investigated the impact of USP7 silencing or pharmacological inhibition on the proliferation and viability of leukemic cell lines. USP7 silencing in HL-60 cells (Supplementary Fig. 1A) significantly reduced cell proliferation (Fig. 1a) by increasing apoptotic cell death (Fig. 1b) and the proportion of cells in the G1 phase (Fig. 1c).

In order to assess the importance of p53 status in this response, USP7 pharmacological inhibition (P22077) was then tested in both HL-60 (p53-) and OCI-AML3 (p53+) cell lines (Fig. 1d-f and g-i, respectively and Supplementary Fig. 1B). This confirmed the results obtained by RNA interference, and indicated that the p53 pathway is in all probability not involved in these USP7 inhibition effects on leukemic cells. As a control of USP7 pharmacological inhibition efficiency, we demonstrated that polyubiquitination of the serine-threonine kinase CHK1, one of the welldescribed USP7 substrates [30, 31], was increased, and that in turn, CHK1 protein level was decreased in both leukemic cell lines (Supplementary Fig. 1C-E).

The effect of USP7 inhibition in vivo was then studied in an AML xenograft model. Briefly, 2 million OCI-AML3 cells were injected into the tail vein of immunodeficient adult NSG mice after pre-conditioning with a sub-lethal treatment of busulfan one day prior to injection in order to establish AML disease. USP7 inhibitor treatment by intraperitoneal injection over a 5-day span significantly improved overall mouse survival $(* * * P=0.0001)$ compared with vehicle-treated mice (Fig. 1j). Altogether, these results show that USP7 inhibition significantly reduces leukemic cells proliferation and impairs their viability in vitro and in vivo.

\section{USP7 inhibition impacts primary AML cell viability without affecting normal cells}

We then performed similar experiments on primary AML cells and normal peripheral blood mononuclear cells (PBMC). As shown in Fig. 2a, USP7 inhibition with P22077 significantly reduced cell viability in three different primary AML samples, while PBMCs were barely affected by the treatment. It should be noted that primary AML and 

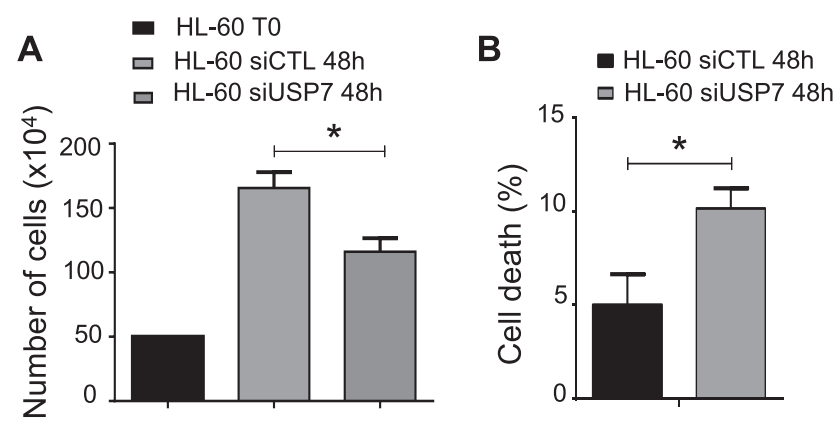

C

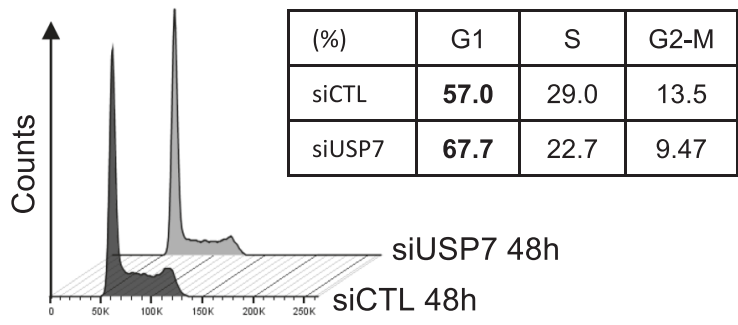

Propidium iodide $(\mathrm{PI})$
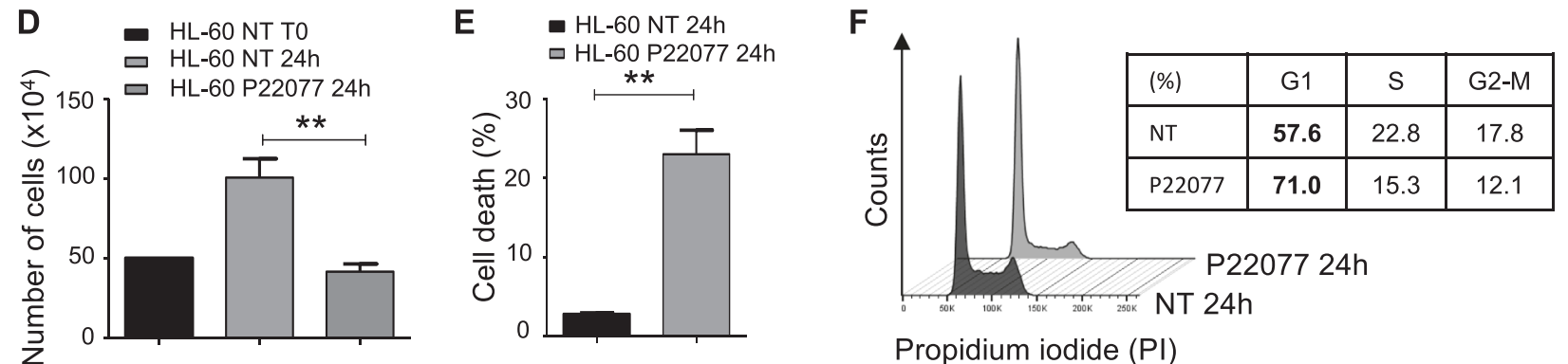

Propidium iodide (PI)

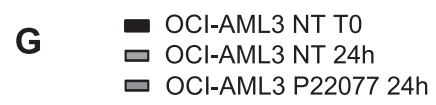

H

I
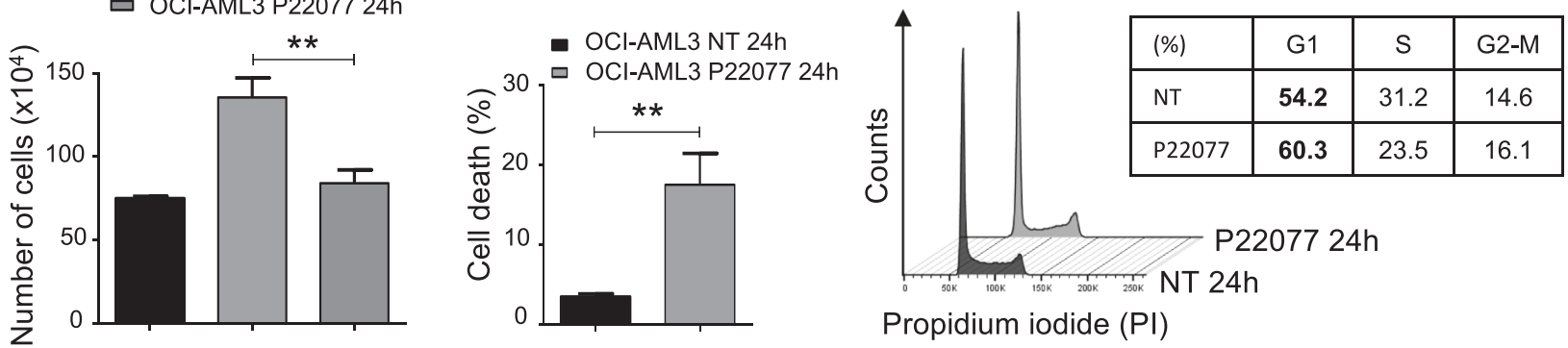

J In vivo OCl-AML3 cell line

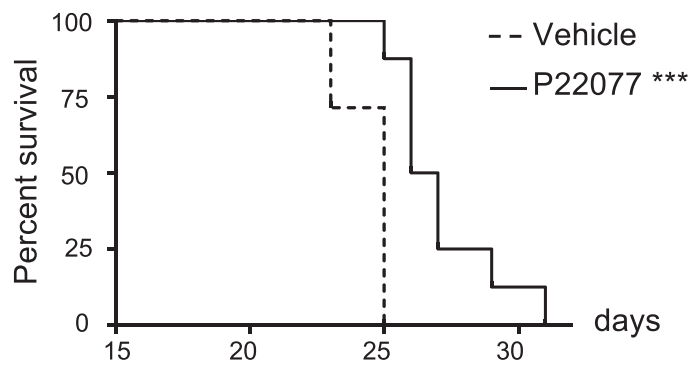

PBMCs classically do not proliferate under these culture conditions. Altogether, these results suggest that USP7 controls leukemic cell viability without affecting normal hematopoietic cell survival.

Subsequently, to study the effect of USP7 inhibition in vivo, we used the PDX model. Briefly, 3 million unsorted primary AML cells were injected into adult NSG mice (6-9 weeks) after pre-conditioning with a sub-lethal treatment of busulfan 1 day prior to injection. Engraftment efficiency was monitored in peripheral blood by flow cytometry analysis of human $\mathrm{CD}_{4} 5^{+}$and $\mathrm{CD} 3^{+}$positive cells at 22 weeks after xenotransplantation (Fig. 2b and Supplementary Fig. 2A) before starting treatment and for cohort randomization. Mice showing at least $20 \mathrm{AML}$ human blasts per microliter of peripheral blood were assigned to experimental groups in order to obtain balanced 
Fig. 1 USP7 Inhibition impacts leukemic cell proliferation and viability in vitro and in vivo. a HL-60 cells were transfected with the control (CTL) or USP7 siRNA for $48 \mathrm{~h}$, cell quantity was then assessed by Trypan Blue staining. For statistical analyses, a nonparametric $t$-test was used. $* P \leq 0.05$ and $* * P \leq 0.01 . n=5$. b In similar experiments to the data in a, cell death was assessed by flow cytometry with annexinV/cell viability staining using a MACSQuant VYB flow cytometer and raw data were analyzed with FlowJo software. Data represent the mean \pm SEM of four experiments. Statistical significance was determined by student's unpaired $t$ test. c In similar experiments as described in a, cell cycle distribution was determined using propidium iodide (PI) staining and analyzed by flow cytometry using a MACSQuant VYB flow cytometer and completed by analyses with FlowJo software. Representative cell cycle distribution profiles from three independent experiments are shown. d HL-60 cells were treated as indicated and $48 \mathrm{~h}$ after cell quantity was assessed by Trypan Blue staining. Statistical analyses were performed as in a $(n=5)$. e In similar experiments to the data in $\mathbf{d}$, cell death was assessed by flow cytometry using annexinV/cell viability staining. Statistical significance was determined as in $\mathbf{b}(n=3)$. $\mathbf{f}$ In similar experiments to the data in $\mathbf{d}$, cell cycle distribution was determined using propidium iodide (PI) staining. Representative cell cycle distribution profiles from three independent experiments are shown. g OCI-AML3 cells were treated as indicated and $48 \mathrm{~h}$ after the number of cells was assessed by Trypan Blue staining. Statistical analyses were performed as in $\mathbf{a}(n=$ 6). $\mathbf{h}$ In similar experiments to the data in $\mathbf{g}$, cell death was assessed by flow cytometry using annexinV/cell viability staining. Statistical significance was determined as in $\mathbf{b}(n=5)$. $\mathbf{i}$ In similar experiments to the data in $\mathbf{g}$, cell cycle distribution was determined using propidium iodide (PI) staining. Representative cell cycle distribution profiles from three independent experiments are shown. $\mathbf{j}$ Kaplan-Meier curves of mice survival were established for NSG mice engrafted with OCIAML3, and treated with P22077 (30 mg/kg/day) or the vehicle (DMSO + corn oil) for 5 days. For statistical analysis, a Mantel-Cox test was used. $* * * P \leq 0.001$.

average engraftment levels in each cohort at the initiation of therapy (Fig. 2c). PDX mice were treated by intraperitoneal injection with $15 \mathrm{mg} / \mathrm{kg}$ P22077, three times per week for 2 weeks and daily for 1 week (Fig. 2b). After 3 weeks of treatment, a significant reduction in total tumor burden in bone marrow and spleen was observed in USP7 inhibitortreated versus untreated mice (Fig. 2d). In addition, a significant reduction in spleen size was monitored in USP7 inhibitor-treated mice compared with untreated mice (Fig. 2e, f). It is important to note that P22077 was used at a well-tolerated dose, which was monitored by mouse body weight and the viability of normal hematopoietic cells in murine bone marrow (Fig. 2g, h). Otherwise, the USP7 inhibitor does not affect normal hematopoietic murine cells in the spleen, as determined by the number of CD45.1positive cells (Supplementary Fig. 2B).

\section{USP7 abundance, transcriptomic gene signature and AML patient outcome}

We then determined USP7 protein levels in a cohort of 57 primary AML samples by western blot analysis. Ten of these samples were not usable (degraded or did not have enough actin signal, annotated as not included $(N I)$ in Fig. 4a). Therefore, we performed the analysis on 47 patient samples. As shown in Fig. 3a, USP7 protein level was highly variable in AML samples. Interestingly, a similar analysis was performed in a panel of six AML cell lines (Supplementary Fig. 3A), and the same heterogeneous expression was shown.

Therefore, we divided the patient data into two groups according to their median USP7 expression: low or high protein expressers (Table 1). There were no significant differences between patients with low and high USP7 protein expression in terms of age, sex, cytogenetics, or NPM1 status (Table 1). We also found that USP7 expression seems to correlate with FLT3-ITD mutation. Furthermore, we also observed that samples with high USP7 expression presented an increase in white blood cells, which is consistent with a frequent clinical observation in AML patients harboring mutations in the FLT3 tyrosine-kinase receptor. Consequently, we investigated the impact of FLT3-ITD on USP7 abundance in two human FLT3-ITD-positive cell lines, MOLM-14 and MV4-11. Pharmacological inhibition of FLT3 activity in these leukemic cells with AC220 (quizartinib), a specific FLT3 inhibitor approved by the US Food and Drug Administration for the treatment of patients with relapse/refractory FLT3-ITD AML, by 2, 6 or $24 \mathrm{~h}$ incubation was not followed by a significant decrease in USP7 level (Supplementary Fig. 3B).

Given our results and that USP7 has been implicated in transcription regulation $[18,21,32]$, we then questioned whether high USP7 abundance AML cells display a specific USP7-related transcriptomic signature. To test this hypothesis, USP7 abundance (transcript and protein) was quantified in AML patient samples. We found that USP7 transcript abundance and USP7 protein levels were highly correlated $(R=0.8073, * * P=0.0085)$ (Fig. $3 b)$. We then defined a USP7-specific gene signature (41 genes upregulated, Supplementary Table 1) from The Cancer Genome Atlas transcriptomic database [25], and found that this signature is highly enriched in the transcriptome of AML patient samples at relapse (HACKL cohort, GEO: GSE66525 [27]) (Fig. 3c). We also observed a similar enrichment in transcriptomes of cytarabine-resistant human AML cells purified from PDX models treated with clinically relevant doses of cytarabine (GEO: GSE97631 [28]) (Fig. 3d). These data highlight a correlation between high USP7 transcriptomic signature, AML cell resistance to chemotherapeutic drugs and relapse. Therefore, USP7 signature could represent a new predictive marker of chemoresistance and relapse in AML.

To further characterize the heterogeneity of primary AML samples, we performed single-cell RNA sequencing of AML cells collected either at diagnosis or at relapse from an AML patient (IM10) (Fig. 3e) treated with a combination 


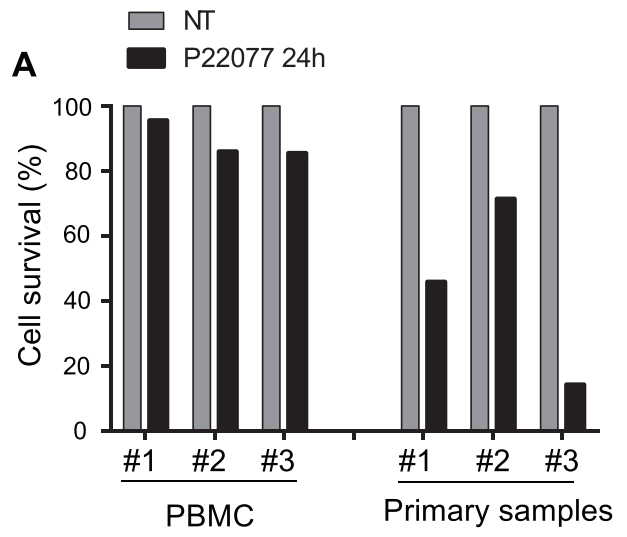

C

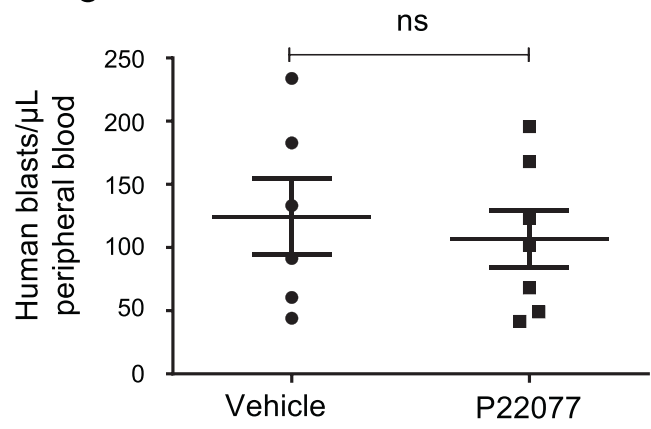

E

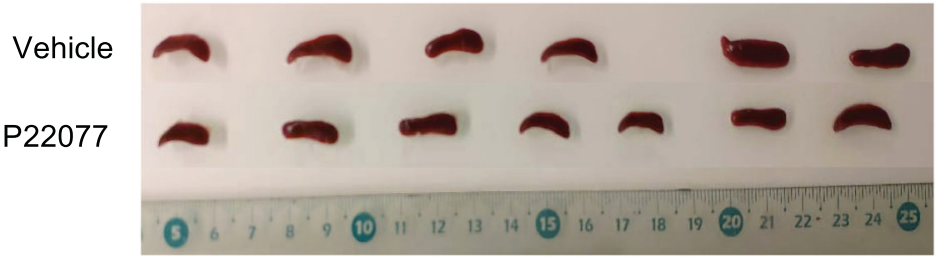

G

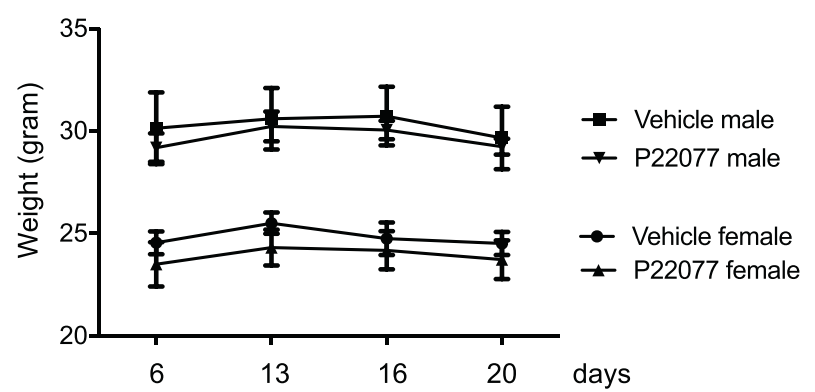

B
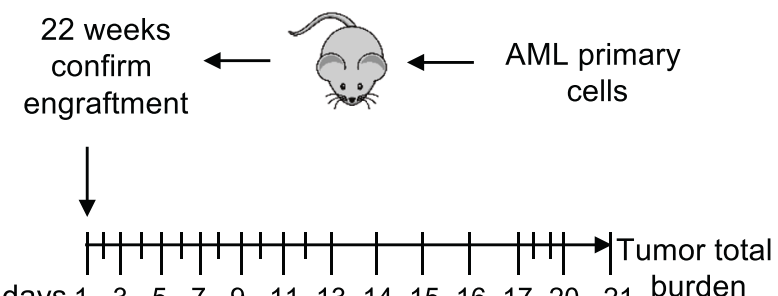

days $1 \quad 3 \quad \begin{array}{lllllllllllll}5 & 7 & 9 & 11 & 13 & 14 & 15 & 16 & 17 & 20 & 21 & \text { burden }\end{array}$

P22077 treatment

$15 \mathrm{mg} / \mathrm{kg}$

D

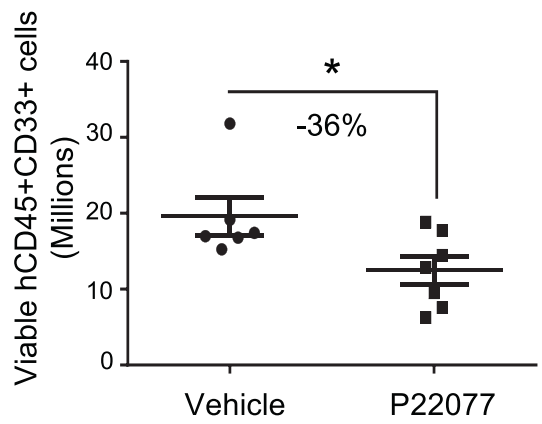

F

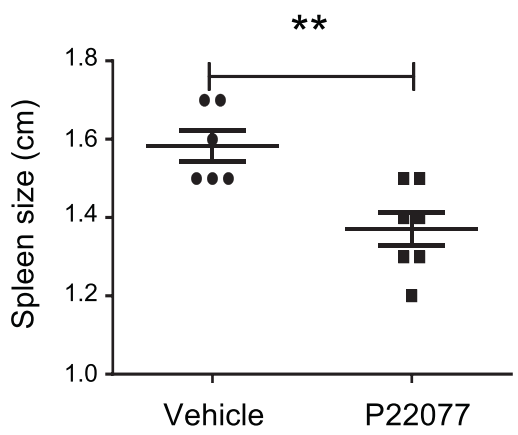

H

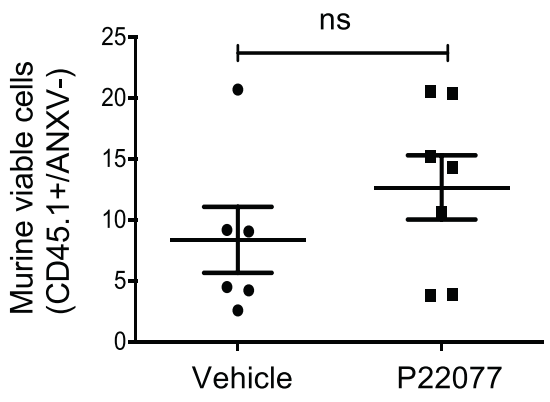

of anthracycline and cytarabine. Based on the difference in their gene expression, we identified two distinct transcriptional clusters of cells at diagnosis and relapse (Fig. 3f), and observed that at relapse, the decrease was striking in cluster
2 compared with cluster 1 (Fig. 3f, right panel), which implies that the signature of cluster 1 corresponds to the gene signature of the chemoresistant cells in this patient. Interrogation of two public transcriptomic datasets 
Fig. 2 USP7 inhibition impacts primary leukemic cell proliferation and viability in vitro and in vivo, without impacting normal cells. a Peripheral blood mononuclear cells (PBMC, $n=3$ ) and AML primary samples $(n=3)$ were treated with $10 \mu \mathrm{M}$ P22077 for $24 \mathrm{~h}$, and cell viability was assessed by flow cytometry with annexinV/cell viability staining, using a MACSQuant VYB flow cytometer and data were analyzed with FlowJo software. b Schematic diagram of the treatment regimen and schedule used to treat NSG-based PDX models with P22077. Peripheral blood engraftment was assessed after 22 weeks and mice were assigned to experimental groups. Six mice were treated with the vehicle (PBS + DMSO) or $15 \mathrm{mg} / \mathrm{kg} /$ day P22077 via intraperitoneal route. After 3 weeks of treatment, mice were sacrificed in order to characterize viable residual AML cells. c Engraftment efficiency was measured in peripheral blood by flow cytometry analysis at 22 weeks after xenotransplantation. Mice with at least 20 human AML blasts/ $\mu \mathrm{l}$ of blood were assigned to experimental groups to obtain balanced average engraftment levels in each cohort at initiation of therapy. Six mice were assigned to the vehicle group and seven mice were assigned to the P22077 treatment group. For statistical analyses, a $t$-test was used, ns not significant. d The total number of viable human AML cells expressing CD45+ and CD33 + was analyzed and quantified using flow cytometry in the bone marrow and spleen of P22077-treated AMLxenografted mice compared with vehicle-treated AML-xenografted mice (total cell tumor burden in millions). For statistical analysis, a nonparametric $t$-test was used. $* P \leq 0.05$. e, f Spleen size was monitored in USP7 inhibitor condition (P22077, $n=7$ ) compared with untreated mice (Vehicle, $n=6$ ). For statistical analysis, a nonparametric $t$-test was used. $* * P \leq 0.01 \mathrm{~g}$ The body weight of the mice was monitored during the experiment. The mean of each group is presented and male and female mice are differentiated. h Murine viable cells were measured by flow cytometry analysis (CD45.1+ ANXV - cells) to determine the toxicity of P22077 in bone marrow. For statistical analyses, a $t$-test was used, ns not significant.

established on diagnosis of AML patients (TCGA Network [25], and Verhaak database GSE6891 [26]) revealed that this chemoresistant gene signature was enriched in AML samples with high USP7 abundance (Fig. 3g, h). USP7 protein abundance was high in primary cells from these patients (Supplementary Fig. 3C), which is consistent with the data described above. These data strongly suggest that high USP7 levels are associated with chemoresistance in AML cells, and with the initiation of relapse following standard chemotherapy treatment. Overall, these data reveal that this high USP7 signature is a predictive marker of resistance and relapse in AML cells.

\section{USP7 and CHK1 protein expression are correlated in AML}

As previously described, CHEKI expression is an independent prognostic marker in AML and the high protein abundance correlates with resistance to cytarabine in primary AML cells [24]. In order to determine whether USP7 participates in chemoresistance of leukemic cells by modulation of CHK1 abundance/stability, we first investigated their protein levels in our cohort of 57 primary AML samples. Both CHK1 and USP7 protein expression levels were heterogeneous in AML cells (Fig. 4a, b). Based on the median expression of $\mathrm{CHK} 1$ protein, we then separated the cohort into two groups of high CHK1- (Fig. 4a, c, exemplified by sample \# 29) and low CHK1- (Fig. 4a and Supplementary Fig. 3D, exemplified by samples \#28 and \#30) expressing samples. A significant correlation between CHK1 and USP7 protein levels was found in high CHK1 (Fig. 4c) but not in the low CHK1 samples (Supplementary Fig. 3D). Overall, these data strongly suggest a functional link between USP7 and CHK1 in leukemic cells that express high CHK1 levels.

\section{USP7 interacts with and stabilizes CHK1 protein through $\mathrm{K} 48$ deubiquitination in AML cells}

To determine whether USP7 can regulate CHK1 in leukemic cells, we first immunoprecipitated CHK1 from HL-60 and HEL leukemic cell lines (Fig. 5a, b, respectively). In both cell lines USP7 co-immunoprecipitated with CHK1. This demonstrates the presence of both proteins in the same complex.

This interaction was further confirmed by PLA used to visualize the proximity between two proteins. Through this approach, we confirmed that CHK1 and USP7 are colocalized in HL-60 cells, as shown by the presence of PLA dots in cells (Fig. 5c). The absence of staining in the presence of a single PLA antibody was confirmed as recommended (Supplementary Fig. 4a). Moreover, CHK1 or USP7 silencing with siRNA significantly reduced the number of PLA dots, which demonstrates the specificity of this signal (Fig. 5c).

Since we demonstrated that USP7 and CHK1 interact in leukemic cells, we then determined whether USP7 can affect CHK1 ubiquitination in AML cells. First, we performed experiments by overexpressing HA-tagged ubiquitin in HL-60 cells and using siRNA against CHK1 or USP7 by treatment with the proteasome inhibitor MG132 to stabilize ubiquitinated forms of the protein. Endogenous CHK1 was then immunoprecipitated and immunoblotted with CHK1 and HA-antibodies. The HA-ubiquitin signal present in immunoprecipitated CHK1 was higher when USP7 was silenced (Fig. 5d, and Supplementary Fig. 4B). To further support this observation, endogenous CHK1 was immunoprecipitated from HL-60 cells treated with P22077 in the presence of the proteasome inhibitor MG132. These fractions were immunoblotted and probed with an anti-ubiquitin antibody. The results indicate that CHK1 was more abundantly ubiquitinated following USP7 inhibition compared with the control, which suggests that USP7 is a negative regulator of CHK1 ubiquitination (Supplementary Fig. 1C). Collectively, these results indicate that USP7 is an important mediator of CHK1 deubiquitination in leukemic cells.

We then investigated whether USP7 can regulate CHK1 protein level or its subcellular localization in leukemic cells. 
Fig. 3 USP7 gene expression and transcriptomic gene set. a USP7 protein levels were determined by immunoblotting in 57 primary AML samples. Actin was used as the loading control and extracts from KGla leukemic cell line served as a between-gel control. Total USP7 protein level quantification is reported. b Linear regression analysis for the correlation between USP7 mRNA level and USP7 protein level, analyzed in nine AML primary samples. c GSEA of the high USP7 gene signature in transcriptomes of patients with AML at relapse (red) compared with at diagnosis (blue). d GSEA of the high USP7 gene signature in transcriptome of patients with AML that are low (red) compared with high (blue) responders in PDX models. e Schematic diagram of primary AML samples from one patient (IM10) at diagnosis and at relapse following chemotherapeutic induction. AML blast cells were sorted based on the CD45+, CD33+ and ANXV - expression and then processed for single-cell $3^{\prime}$ RNA sequencing analysis. f SNE plot of scRNAseq data with cells colored according to $\mathrm{K}$-means cluster assignment of the cells from IM10 at diagnosis, and at relapse. $\mathbf{g}$ Gene set enrichment analysis (GSEA) of cluster 1 signature generated using the cells at diagnosis and at relapse was performed from the transcriptomes of the TCGA database. $\mathbf{h}$ The same analysis as in $\mathbf{g}$ was performed from the transcriptome of the Verhack database.
A

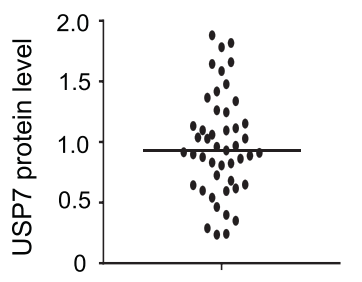

C High USP7 signature (41 genes)

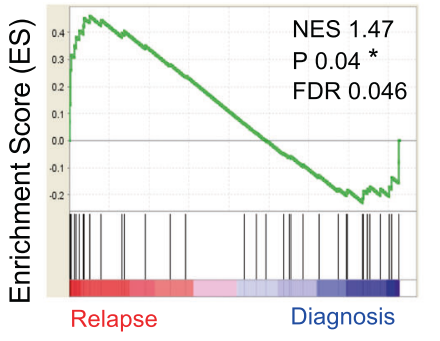

E
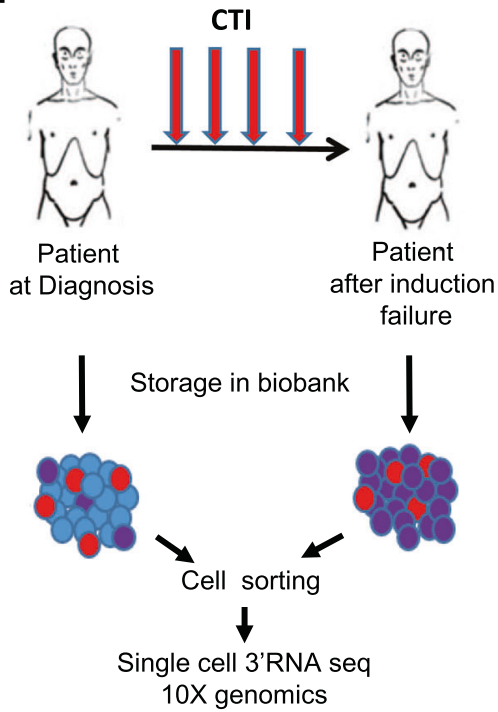

G

In TCGA data base

Signature UP Cluster 1 (220 genes)

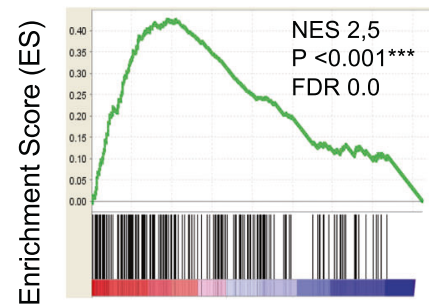

High USP7 patients

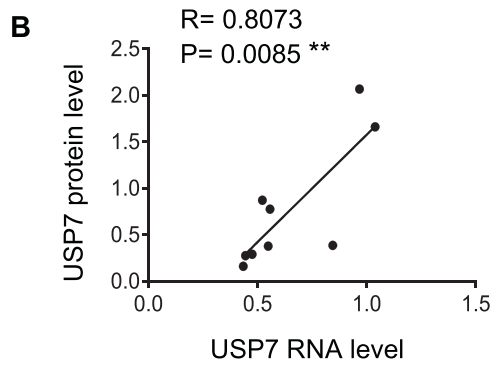

D High USP7 signature (41 genes)

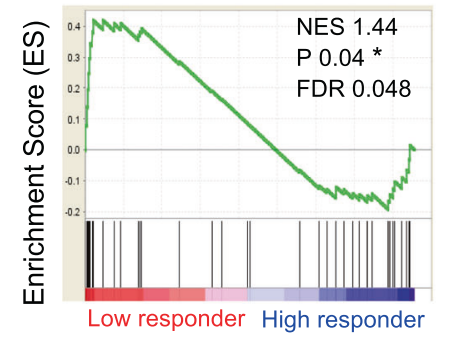

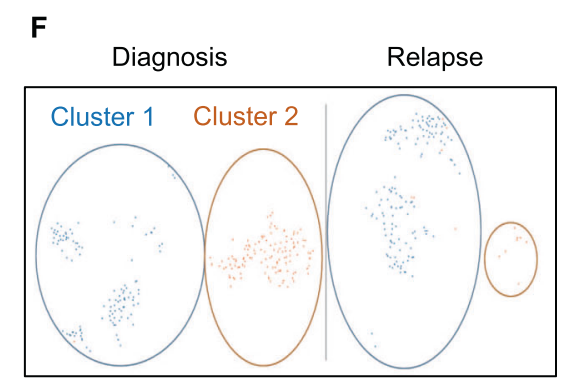

H

In Verhack data base Signature UP Cluster 1 (220 genes)

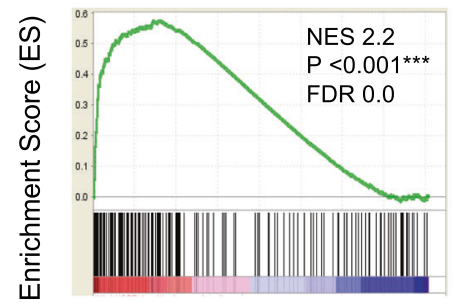

High USP7 patients

Having performed USP7 pharmacological inhibition in HL-60 (p53-) and OCI-AML3 (p53+) (Supplementary Fig. 1D, E), we went further and silenced USP7 expression by RNA interference in HL-60 and HEL (p53+) cell lines, and observed a significant decrease in CHK1 protein levels (Supplementary Fig. 4C and 4D, respectively), although only half of the USP7 proteins were downregulated with these two siRNA sequences in HL-60. Using immunofluorescence, we 
Table 1 Biological properties of primary AML samples. A summary of the main biological properties of primary AML samples used in this study.

\begin{tabular}{|c|c|c|c|c|c|c|}
\hline & \multirow{3}{*}{ Total } & \multicolumn{4}{|c|}{ USP7 expression } & \multirow{3}{*}{$P$ value } \\
\hline & & \multicolumn{2}{|c|}{$\begin{array}{l}\text { Low } \\
(<\text { median value })\end{array}$} & \multicolumn{2}{|c|}{$\begin{array}{l}\text { High } \\
\text { (>median value) }\end{array}$} & \\
\hline & & & $n$ & & $n$ & \\
\hline Age in years, mean $\pm \mathrm{SD}, n=46$ & $56.26 \pm 17.69$ & 23 & $56.00 \pm 20.58$ & 23 & $56.52 \pm 14.71$ & 0.922 \\
\hline \multirow{2}{*}{$\begin{array}{l}\text { White blood cells in } \mathrm{g} / \text { liter, mean } \pm \mathrm{SEM} \text {, } \\
n=44\end{array}$} & $62.81 \pm 10.92$ & 21 & $44.07 \pm 12.63$ & 23 & $79.94 \pm 16.90$ & 0.101 \\
\hline & Total & $n$ & $\%$ & $n$ & $\%$ & $P$ value \\
\hline Gender, $n(\%)$ & & & & & & 0.750 \\
\hline Male & $32(69.6)$ & 17 & (73.9) & 15 & $(65.2)$ & \\
\hline Female & $14(30.4)$ & 6 & $(26.1)$ & 8 & $(34.8)$ & \\
\hline Secondary AML, $n(\%)$ & & & & & & 0.021 \\
\hline No & $38(88.4)$ & 16 & $(76.2)$ & 22 & $(100)$ & \\
\hline Yes & $5(11.6)$ & 5 & $(23.8)$ & 0 & (0) & \\
\hline Cytogenetic risk, $n(\%)$ & & & & & & 0.114 \\
\hline Favorable & $6(13.0)$ & 4 & $(17.4)$ & 2 & $(8.7)$ & \\
\hline Intermediate & $37(80.4)$ & 16 & $(69.6)$ & 21 & $(91.3)$ & \\
\hline Adverse & $3(6.5)$ & 3 & $(13.0)$ & 0 & (0) & \\
\hline NPM1 mutation, $n(\%)$ & & & & & & $>0.999$ \\
\hline No & $22(51.2)$ & 10 & (50) & 12 & $(52.2)$ & \\
\hline Yes & $21(48.3)$ & 10 & (50) & 11 & $(47.8)$ & \\
\hline FLT3-ITD mutation, $n(\%)$ & & & & & & 0.003 \\
\hline No & $25(56.8)$ & 17 & $(80.9)$ & 8 & $(34.8)$ & \\
\hline Yes & $19(43.2)$ & 4 & $(19.0)$ & 15 & $(65.2)$ & \\
\hline
\end{tabular}

Statistical analysis was established by the unpaired $t$-test for age and white blood cell count and then a Fisher's exact tests were applied to determine the $P$ value. also confirmed that $\sim 50 \%$ of the CHK1 protein signal disappears after $24 \mathrm{~h}$ of USP7 inhibition, without any changes in CHK1 subcellular localization (Supplementary Fig. 4E). Together, these experiments show that genetic or pharmacological inhibition of USP7 decreases CHK1 protein levels in three different leukemic cell lines, independently of p53 status, which demonstrates the role of USP7 deubiquitinase activity on CHK1 stability.

To complete our investigation, experiments were performed to test whether the half-life of CHK1 is regulated by USP7 in leukemic cells. To this end, HL-60 cells were treated with P22077 and the half-life of CHK1 was measured in the presence of $50 \mu \mathrm{g} / \mathrm{ml}$ cycloheximide (CHX) to inhibit protein biogenesis. As shown in Fig. 5e, USP7 inhibition significantly shortened the half-life of CHK1 protein after $6 \mathrm{~h}$ of P22077 treatment, without affecting CHEK1 mRNA expression (Supplementary Fig. 4F).

Finally, to further understand which poly-ubiquitin chain on CHK1 is removed by USP7, we immunoprecipitated an equal amount of K48-linked poly-ubiquitin or K63 linked poly-ubiquitin proteins with specific antibodies in HL-60 cells. The results indicated that K48-linked ubiquitin species conjugated on CHK1 represent the major form of ubiquitin moieties (Fig. 5f). Moreover, inhibition of USP7 by P22077 resulted in a significant increase in the K48 poly-ubiquitylation of CHK1 in HL-60 cells (Fig. 5g). Overall, our data demonstrate that USP7 stabilizes CHK1 by preventing proteasomal degradation by removing K48linked poly-ubiquitin chains in leukemic cells.

On the whole, our results predict that targeting USP7 in leukemic cells, which exhibit a strong dependence on CHK1 level for proliferation [24], can impair their survival.

\section{Targeting USP7 overcomes cytarabine resistance in AML}

To explore and confirm whether USP7 participates in the chemoresistance of leukemic cells, we examined the capacity to sensitize HL-60 and OCI-AML3 cells to cytarabine (AraC) treatment through USP7 inhibition. As shown in Fig. 6a, b, P22077 synergistically (Supplementary Fig. 4G) enhanced cell death induced by cytarabine in these resistant cell lines in vitro, independently of p53 status.

To further support our hypothesis, we quantified the capacity of primary AML cells to form colonies in a methylcellulose-based semi-solid medium, when exposed to clinically relevant concentrations of cytarabine $(10 \mathrm{nM})$ combined with the P22077 inhibitor (Fig. 6c, d). Based on the median expression of USP7 protein, we separated this cohort into two groups of high USP7 and low USP7 expressing samples. Leukemic cells with high USP7 levels (Fig. 6c) tended to be more resistant to cytarabine compared with cells that expressed lower levels of USP7 (Fig. 6d), which is consistent with our transcriptomics analyses 
A

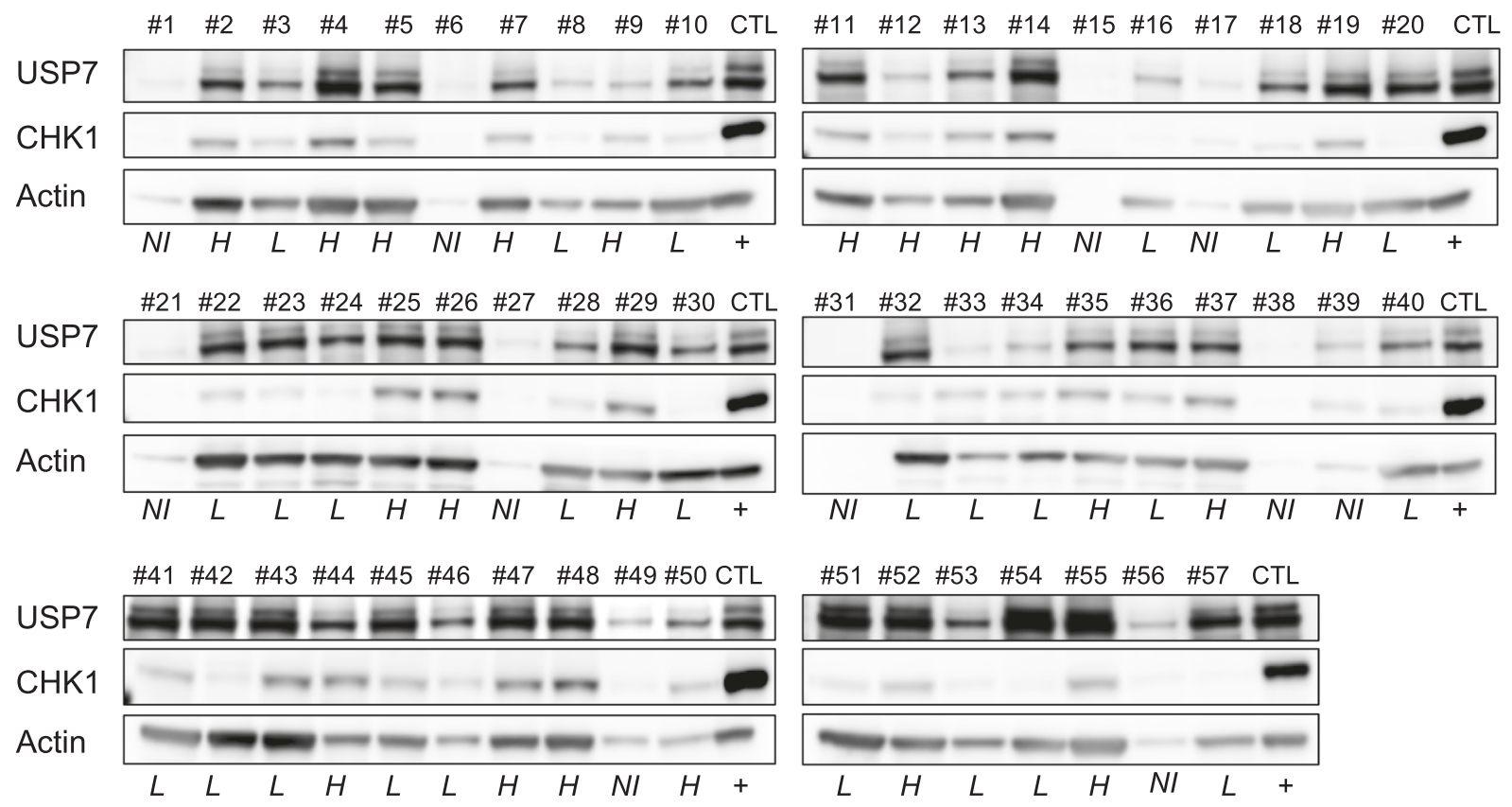

B

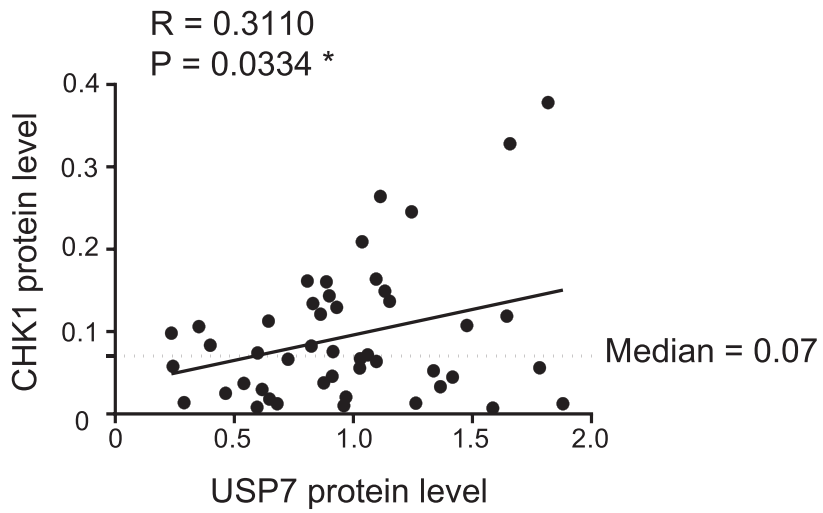

C

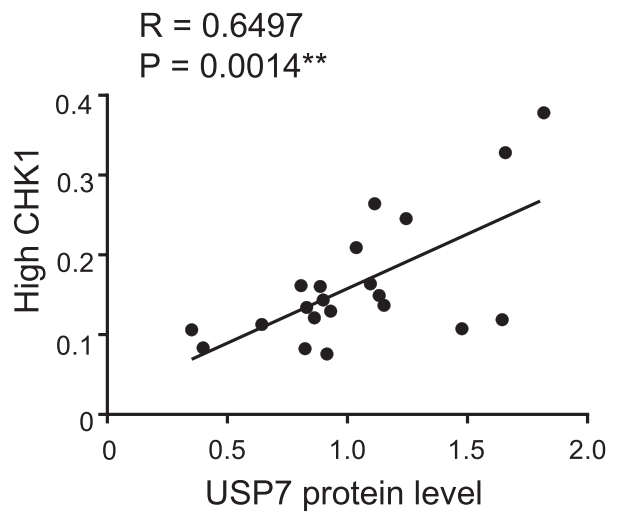

Fig. 4 USP7 and CHK1 protein expression correlate in primary AML samples. a CHK1 and USP7 protein levels were determined by immunoblot and actin was used as a loading control in 46 primary AML samples. KG1a cell line extract was used as an internal control between gels (CTL). Samples were considered "high CHK1

abundance" if the average protein abundance value was higher than the median. b Linear regression analysis for the correlation between CHK1 and USP7 protein levels in primary AML samples. c Same analysis as in b with 21 primary AML samples with high CHK1 abundance.

(Fig. 3). It is striking to note that USP7 inhibition potentiated the effects of cytarabine in cells that expressed high levels of USP7, leading to reduced colony formation, while it did not modify the sensitivity to cytarabine of cells that expressed low levels of USP7. Our results indicate that P22077 significantly enhances the cytotoxic effect of cytarabine on leukemic cells by reducing their colony-forming potential. In order to determine whether USP7 participates in the chemoresistance of leukemic cells by modulation of CHK1 stability, we then monitored the CHK1/USP7 association by PLA in AML cells treated with cytarabine. A significant increase in the proximity between the two proteins was visualized in cytarabinetreated HL-60 cells (Fig. 6e, f). The specificity of the interaction was confirmed by using a USP7 inhibitor (P22077) that induced a significant decrease in CHK1 protein level (Supplementary Fig. 4E), dramatically reducing the number of PLA dots per cell (Fig. 6e, f). Similar results were obtained with the combination of cytarabine and P22077. Altogether, these data suggest that USP7 plays a role in promoting CHK1 stability in response to DNA-damaging treatment. 
A

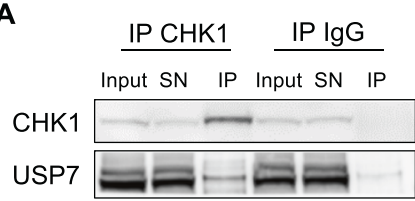

B

Input SN IPCHK1 SiRNA 24h CTL CHK1 CTL CHK1 CTL CHK1 CHK1 USP7

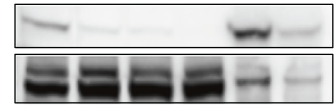

D
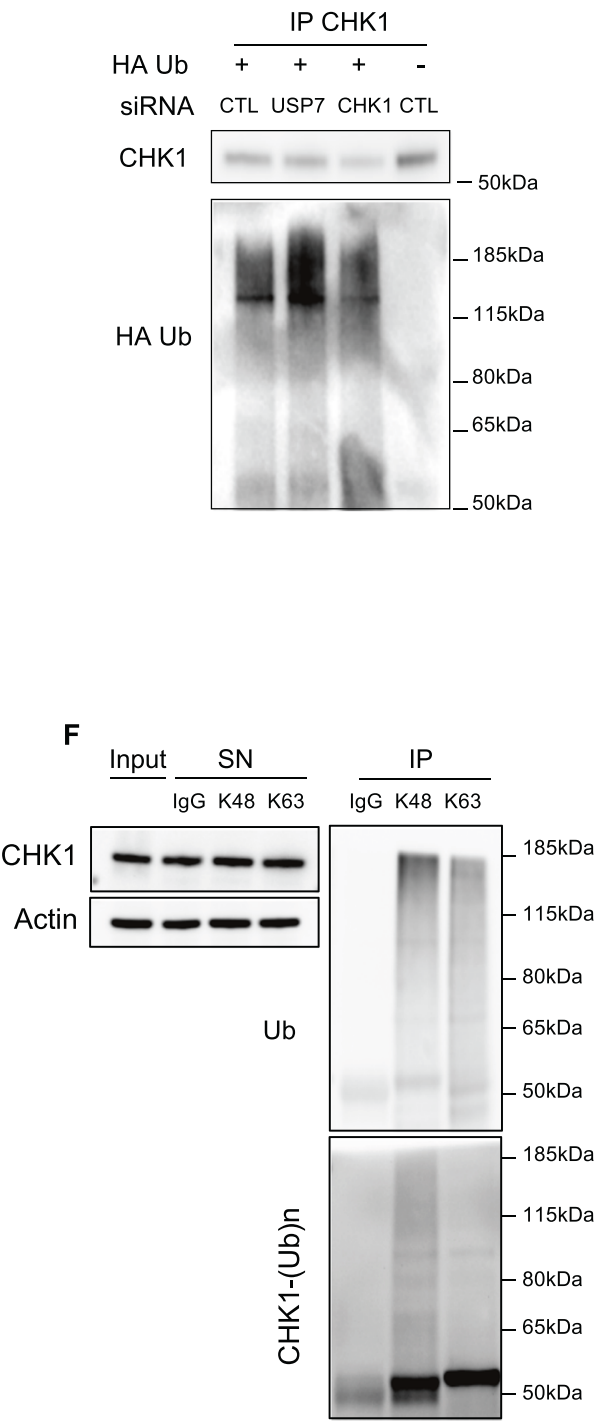

Consequently, we further examined whether a USP7/ CHK1 axis is involved in AML cell chemoresistance by measuring cell death in response to CHK1 inhibitor (SCH900776) treatment alone or in combination with

E

G

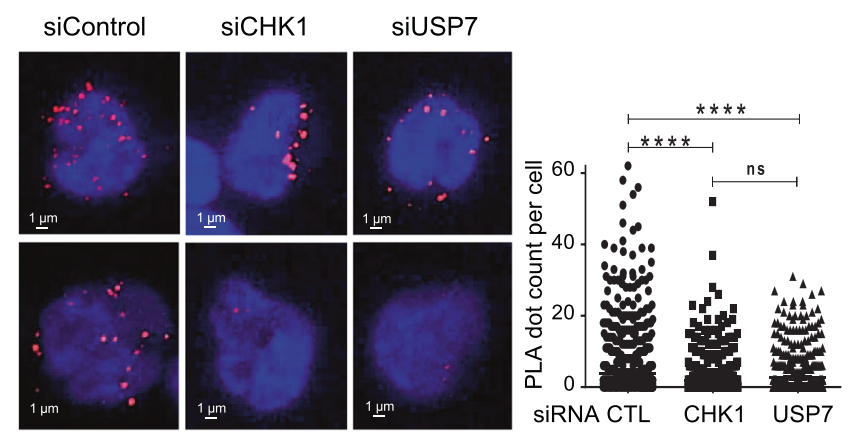

$\mathrm{CHX}$

$(50 \mu \mathrm{g} / \mathrm{ml}) \quad$ T0 $\quad$ T1 $\quad$ T2 $\quad$ T4 $\quad$ T6

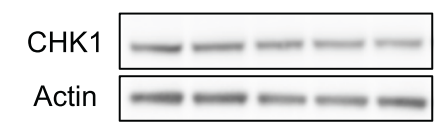

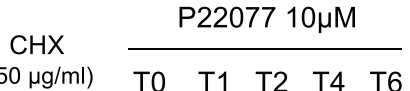
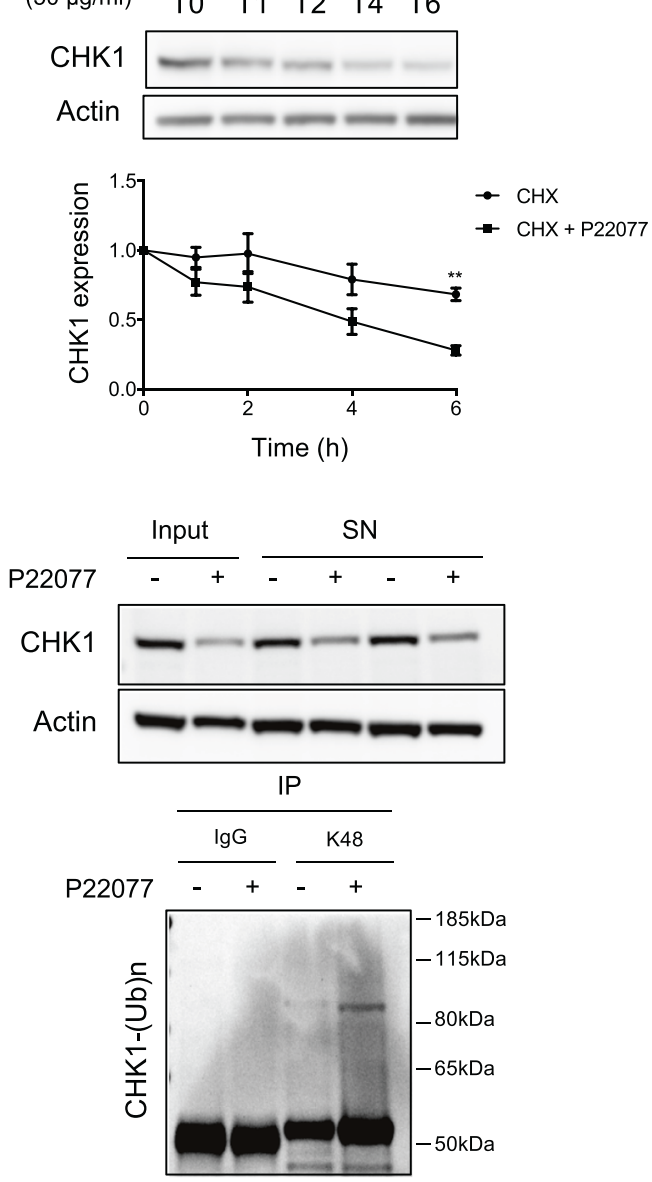

cytarabine and P22077 (Fig. 6g). As described earlier (Fig. 6a, b), P22077 significantly increased cell death after cytarabine treatment. Interestingly, the addition of SCH900776 in P22077 and cytarabine-treated cells did 
Fig. 5 USP7 interacts and deubiquitinates CHK1 in leukemic cells. a Whole-cell lysates from HL-60 cells were immunoprecipitated with anti-CHK1 antibody or an irrelevant Immunoglobulin (IgG) and then immunoblotted with antibodies against the indicated proteins. b A similar experiment to a was performed with anti-CHK1 antibody from siRNA control (CTL) or siRNA against CHK1 transfected HEL cells for $24 \mathrm{~h}$. c Proximity ligation assays were performed using the CHK1 and USP7 antibodies on HL-60 cells transfected with the indicated siRNA for $48 \mathrm{~h}$. DNA was counterstained with DAPI. Z-stack images were acquired by confocal microscopy. Scale bar, $1 \mu \mathrm{m}$. The quantification of foci per cell is presented. Quantification of the images was performed with ZEN and ImageJ software. For co-staining of CHK1 and USP7: mean $=3.75 \pm 0.22$ foci/cell from 1180 nuclei analyzed under siRNA control condition $(n=4)$, mean $=1.85 \pm 0.12$ foci/cell from 1126 nuclei analyzed under siRNA CHK1 condition $(n=4)$, and mean $=2.69 \pm 0.21$ foci/cell from 650 nuclei analyzed under siRNA USP7 condition $(n=3) . \quad P$ values were determined using the Mann-Whitney test, with $* * * * P \leq 0.0001$. d HL-60 cells were transfected with HA-Ub. After $72 \mathrm{~h}$, a new transfection round was performed with siRNA control (CTL) or siRNA against CHK1 or USP7 for another $48 \mathrm{~h}$. Finally, $6 \mathrm{~h}$ before harvesting, cells were treated with $10 \mu \mathrm{g} / \mathrm{ml}$ MG132. Cell extracts were immunoprecipitated with antiCHK1 antibody followed by western blot analysis with the indicated antibodies. e HL-60 were treated with $50 \mu \mathrm{g} / \mathrm{ml}$ cycloheximide (CHX) in the presence or absence of $10 \mu \mathrm{M}$ P22077. Lysates were prepared at the indicated times. Representative western blot is presented and quantification from three independent experiments is shown graphically below. $* * P \leq 0.01$. f HL-60 cellular extracts were prepared for immunoprecipitation (IP) with the indicated antibodies followed by western blotting with anti-CHK1 or anti-ubiquitin antibody. g HL-60 cells were treated with or without $10 \mu \mathrm{M}$ P22077 for $24 \mathrm{~h}$. Six hours before being harvested, cells were treated with $10 \mu \mathrm{g} / \mathrm{ml} \mathrm{MG132}$ and processed for immunoprecipitation (IP) with an irrelevant immunoglobulin (IgG) or an anti-ubiquitin (Lys48-specific) antibody, followed by immunoblotting analysis using anti-CHK1 antibody.

not increase cell death, which suggests that P22077induced cell death is mediated by the USP7/CHK1 axis. Despite the large spectrum of USP7 substrates, we showed that the cytotoxic effect caused by USP7 inhibition is mediated, at least in part, by a decrease in CHK1 expression. Overall, these results indicate that P22077 could be a relevant anti-leukemic drug in overcoming chemoresistance.

Finally, to further understand the molecular mechanisms underlying these effects, we explored the contribution of this USP7/CHK1 axis in the DNA replication fork progression in AML cells. We used the DNA fiber spreading assay for this purpose, in keeping with previous reports that clearly documented the role of USP7 or CHK1 in the DNA replication process [24, 33, 34]. HL-60 cells were cultured in the presence of a USP7 inhibitor (P22077), a CHK1 inhibitor (SCH900776), or a combination of both for $16 \mathrm{~h}$, then the thymidine analogs CldU and IdU were sequentially pulsed for an equal amount of time (20 min each) resulting in the labeling of nascent DNA tracks in vivo. With this method, we monitored replication fork progression as indicated by the presence of nascent DNA at the level of individual replicating DNA molecules. A reduction in nascent DNA track length is indicative of stalled DNA replication. USP7 or CHK1 inhibition led to a significant and similar reduction in the rate of ongoing replication forks in HL-60 cells (Fig. 6h). Interestingly, concomitant inhibition of CHK1 and USP7 did not further increase the replication fork blockage, which supports the hypothesis that USP7 and CHK1 are in the same signaling pathway of DNA replication progression in HL-60 cells.

\section{Discussion}

Recent studies have reported that the USP7 level is elevated in several types of tumors, and that USP7 overexpression is often predictive of a poor prognosis [9, 32, 35, 36]. Several studies consequently suggested USP7 as an attractive pharmaceutical target for various cancers, and pharmacological inhibitors of this enzyme have recently been developed [9, 13-19]. In this study, we showed that USP7 plays a key role in AML chemoresistance. Furthermore, we observed heterogeneous USP7 levels in AML cell lines and primary samples and established that patients with a high USP7 signature were more prone to chemoresistance and relapse. Experiments using single-cell analysis support these findings by showing that the resistant gene signature is enriched in AML samples that exhibit high USP7 levels, which reveals that this chemoresistant cell population preexists at diagnosis and is enriched following first line treatment. Consequently, these data suggest that USP7 could be a new prognostic marker and a potential therapeutic target for AML. Our finding that USP7 inhibition in vitro and in vivo significantly suppresses leukemic cell growth, alone and in combination with chemotherapy, independently of p53 status supports this notion. Although p53 can be stabilized by USP7 [8], it appears that in AML, which does not frequently present p53 mutations, cells do not rely on p53 stabilization. Despite the large spectrum of USP7 substrates, we showed that the cytotoxic effect caused by USP7 inhibition is mediated, at least in part, by a decrease in CHK1 function due to similar anti-leukemic effects with the CHK1 inhibitor SCH900776. Our data clearly demonstrate that the USP7/CHK1 axis plays an important part in DNA replication progression and leukemic proliferation and survival. Based on our recent study in which we showed that high CHK1 protein levels contribute to cellular resistance to cytarabine, in part by facilitating fork progression or stalled fork restart [24], and based on the present results, we now suggest that high CHK1 levels, stabilized by USP7, allow cells to survive by enabling them to adapt to DNA replication stress induced by cytarabine treatment. Therefore, we believe that dysregulation of a USP7/CHK1 axis may be a new "Achilles heel" in AML 
A $\mathrm{HL}-60$ cell line ( $p 53-)$

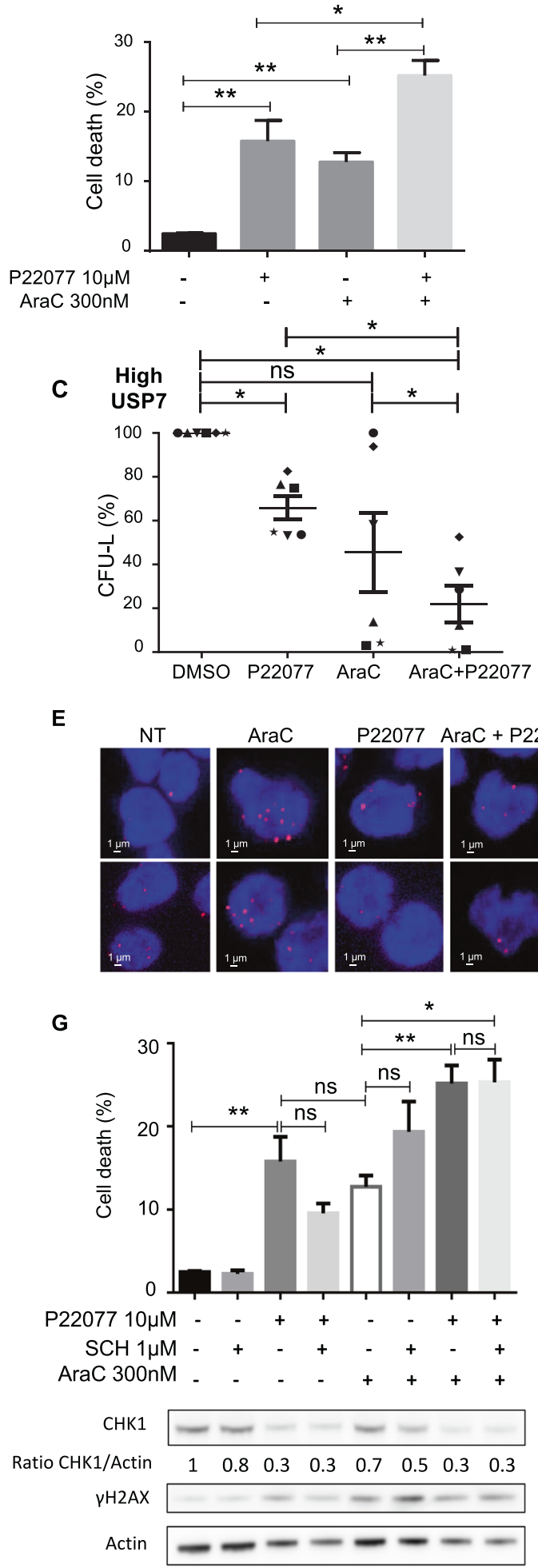

B OCI-AML3 cell line (p53+)
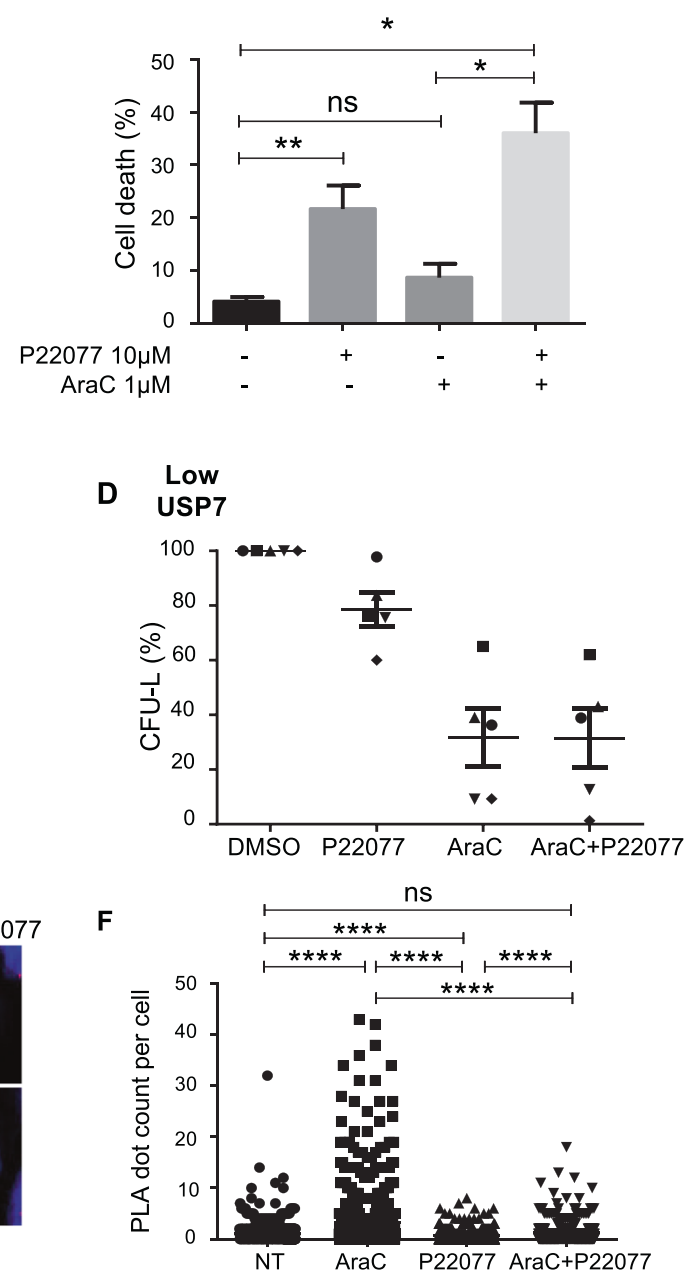

H

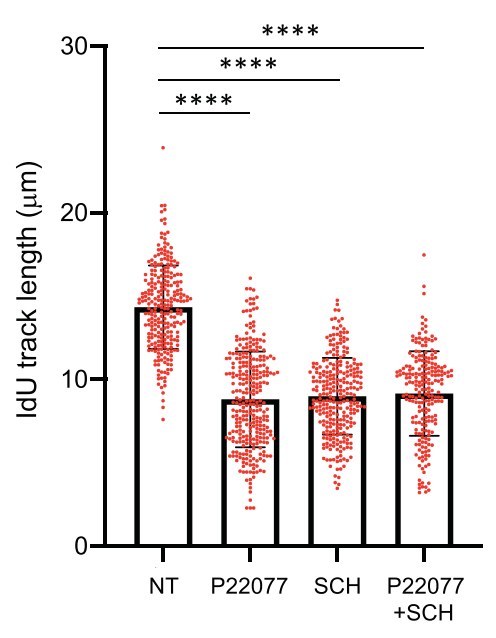

cells. In fact, USP7 inhibition has a profound impact on proliferation and viability in leukemic cells that express high USP7 levels compared with cells that express low
USP7 levels, or in PBMC. This characteristic could provide a useful therapeutic window through which this class of inhibitors could be proposed for clinical trials. 
Fig. 6 USP7 inhibition potentiates cytarabine treatment in AML cells. a HL-60 cells were treated with P22077 $(10 \mu \mathrm{M})$, cytarabine (AraC, $300 \mathrm{nM}$ ) or a combination of both drugs, for $24 \mathrm{~h}$. Cell death was assessed by flow cytometry with annexinV/cell viability staining using a MACSQuant VYB flow cytometer and FlowJo software. Statistical analysis was performed by unpaired $t$-test $(n=4)$. $* P \leq 0.05$ and $* * P \leq 0.01$. b OCI-AML3 cells were treated as indicated for $24 \mathrm{~h}$. Cell death was assessed as in $\mathbf{a}$. Statistics were established by unpaired $t$-test $(n=3) . * P \leq 0.05, * * P \leq 0.01$ and ns not significant. c, d Analyses of the clonogenic properties of high USP7 abundance $(n=6$ primary samples, left panel) and low USP7 abundance ( $n=5$ primary samples, right panel) in AML blast cells with continuous exposure to $10 \mathrm{nM}$ cytarabine (AraC) alone or in combination with $5 \mu \mathrm{M}$ USP7 inhibitor (P22077). Colony formation was assessed after 7 days and represented as the ratio of the number of clones between untreated and treated conditions. For statistical analyses, an unpaired Wilcoxon test was used. $* P \leq 0.05$, and ns not significant. e Proximity ligation assays were performed using anti-CHK1 and anti-USP7 antibodies in HL-60 cells treated with $10 \mu \mathrm{M}$ of P22077 and/or $300 \mathrm{nM}$ of cytarabine for $16 \mathrm{~h}$. DNA was counterstained with DAPI. Z-stack images were acquired by confocal microscopy. f Quantification of PLA foci from three independent experiments was performed with ZEN and ImageJ software. For co-staining of CHK1 and USP7: mean $=1.07 \pm 0.07$ foci/cell from 677 nuclei analyzed for the untreated condition (NT); mean $=3.31 \pm 0.21$ foci/cell from 743 nuclei analyzed for cytarabine treatment; mean $=0.68 \pm 0.04$ foci/cell from 604 nuclei analyzed for P22077 treatment and mean $=1.24 \pm 0.10$ foci/cell from 407 nuclei analyzed for combined treatment. $P$-values were determined using the unpaired $t$-test, $* * * * P<0.0001$. g HL-60 cells were treated with the indicated treatment for $24 \mathrm{~h}$. Cell death was assessed by flow cytometry with annexinV/cell viability staining using a MACSQuant VYB flow cytometer and FlowJo software. Statistical analysis was established by unpaired $t$-test $(n=4) . * P \leq 0.05$ and $* * P \leq 0.01$. In parallel, cellular extracts were collected and expression of the indicated proteins was assessed by western blotting (see under the graph). A representative western blot is presented and ratios for CHK1 protein level normalized to actin loading control are indicated below. $\mathbf{h}$ DNA fiber assay analysis showing iododeoxyuridine (IdU) track length $(\mu \mathrm{m})$. HL60 cells were treated with $10 \mu \mathrm{M}$ of P22077 or $1 \mu \mathrm{M} \mathrm{SCH900776} \mathrm{or}$ the combined treatment for $16 \mathrm{~h}$ and were then labeled with CldU and IdU for 20 min. Mean $( \pm S D)$ track lengths. Data are based on two independent experiments and a total of $260(\mathrm{NT}$; mean $=14.34 \pm$ 2.50), 299 (P22077; mean = 8.81 \pm 2.87$), 278$ (SCH900776; mean $=$ $8.99 \pm 2.30$ ) and 215 (combined treatment; mean $=9.16 \pm 2.54$ ) fibers were scored. Statistical analysis was performed using the unpaired $t$-test with the Welch's correction, $* * * * P<0.00001$.

Although we documented the heterogeneity of USP7 abundance in primary AML samples, the mechanisms by which USP7 protein and mRNA expressions are controlled in leukemic cells remain to be defined. It will be important to investigate the mechanisms that control USP7 expression in the context of AML heterogeneity. In-depth analyses of the clinical and biological characteristics of AML patients revealed that USP7 expression significantly correlates with FLT3-ITD mutation. However, in our studies, we did not observe direct regulation of USP7 expression by the FLT3 tyrosine-kinase receptor, but this specific aspect will require further investigations in the future. Nevertheless, we established that USP7 transcript and protein abundance are highly correlated in AML samples, which suggests that the majority of USP7 regulation might occur at the transcriptional level. Previous reports have documented the transcriptional regulation of USP7, for instance in T-cell acute lymphoblastic leukemia (T-ALL) in which the transcription factor NOTCH1 induces USP7 gene expression, and where USP7 controls the stability of NOTCH1 and the JMJD3 histone demethylase through a positive regulatory loop $[37,38]$. It is noteworthy that the NOTCH-signaling pathway has been reported to play oncogenic and tumor suppressor functions in the hematopoietic system, although the biological and clinical relevance remains unclear in AML [39]. A potential regulatory and functional link between USP7 and NOTCH1 is supported by our transcriptomic analyses considering that Delta-like non-canonical Notch ligand 1 (DLK1) was upregulated in cells enriched in USP7 gene signature. $D L K 1$ encodes the NOTCH activator Delta1 which is involved in primary and model AML cell growth [40]. Furthermore, previous analyses show a significantly shorter survival of patients with high NOTCH1 or Delta1 expression, suggesting that activation of the $\mathrm{NOTCH}$ pathway may be associated with a poor prognosis in AML [41, 42]. All these studies, combined with our data, suggest the existence of a NOTCH/USP7/CHK1 axis involved in AML chemoresistance, which should be investigated in future studies.

Finally, the large spectrum of USP7 substrates and its implication in fundamental cellular and developmental processes define USP7 as a highly important molecule. Depending on the targeted substrates and the cellular context, USP7 might be either a tumor-suppressive or an oncogenic protein. Consequently, it will be important to understand how the acute leukemic cells highjack USP7 functions.

In summary, our study provides evidence that USP7 is an important component in chemoresistant AML cells. Moreover, a combination of a USP7 inhibitor with cytarabine induces synergistic anti-leukemic activity. Overall, our study opens the possibility that targeting USP7 is a promising therapeutic strategy in treating AML and supports the inclusion of USP7 inhibitors in clinical trials aimed at overcoming chemoresistance in AML.

Acknowledgements We would like to sincerely thank Thomas Farge for his technical support during in vivo experiments and Alison Boutet for technical assistance. We thank Laetitia Ligat and Dr Marie Tosolini at the CRCT microscopy facility and Manon Farcé at the CRCT cytometry facility for their helpful discussions of the analyses and the development of macro used in the ImageJ software. We gratefully acknowledge Dr Ze'ev Ronai for the critical review of the manuscript, and sincerely thank all members of the "Cell Cycle and Cancer" team for their critical review during the entire study. The authors sincerely thank Chloé Laplagne for the technical PBMC preparation and Dr Marie Jeanne Pillaire for insightful discussions on DNA fiber spreading experiments. Maëlle Cartel is a recipient of a fellowship from the Ligue Nationale contre le Cancer. We are grateful to our healthcare professionals for their boundless investment during the COVID-19 crisis. 
Author contributions MC, PLM, MG, LD, JES, SM, and CD conceived and designed the study; MC, PLM, MG, LD, and CD performed experiments; SB and VMDM. contributed clinical samples; $\mathrm{MC}, \mathrm{AB}, \mathrm{JES}, \mathrm{SM}$, and CD wrote the manuscript; AB, JES, SM, and $\mathrm{CD}$ insured administrative, technical and material support; SM and CD supervised the project.

\section{Compliance with ethical standards}

Conflict of interest The authors declare that they have no conflict of interest.

Publisher's note Springer Nature remains neutral with regard to jurisdictional claims in published maps and institutional affiliations.

\section{References}

1. Döhner H, Weisdorf DJ, Bloomfield CD. Acute myeloid leukemia. N Engl J Med. 2015;373:1136-52.

2. Papaemmanuil E, Gerstung M, Bullinger L, Gaidzik VI, Paschka $\mathrm{P}$, Roberts ND, et al. Genomic classification and prognosis in acute myeloid leukemia. N Engl J Med. 2016;374:2209-21.

3. Yang M, Zhao J, Liu T, Yang X, Wei H, Xu W, et al. Use of FLT3 inhibitors in acute myeloid leukemia remission induction or salvage therapy: systematic review and meta-analysis. Cancer Manag Res. 2018;10:2635-52.

4. DiNardo CD, Stein EM, de Botton S, Roboz GJ, Altman JK, Mims AS, et al. Durable remissions with ivosidenib in IDH1mutated relapsed or refractory AML. N Engl J Med. 2018; 378:2386-98.

5. Zhou J, Chng WJ. Resistance to FLT3 inhibitors in acute myeloid leukemia: molecular mechanisms and resensitizing strategies. World J Clin Oncol. 2018;9:90-7.

6. Harrigan JA, Jacq X, Martin NM, Jackson SP. Deubiquitylating enzymes and drug discovery: emerging opportunities. Nat Rev Drug Discov. 2018;17:57-78.

7. Basu B, Saha G, Choudhury SG, Ghosh MK. Cellular homeostasis or tumorigenesis: USP7 playing the double agent. Cancer Cell Microenviron. 2017;4:e1624.

8. Li M, Brooks CL, Kon N, Gu W. A dynamic role of HAUSP in the p53-Mdm2 pathway. Mol Cell. 2004;13:879-86.

9. Bhattacharya S, Chakraborty D, Basu M, Ghosh MK. Emerging insights into HAUSP (USP7) in physiology, cancer and other diseases. Signal Transduct Target Ther. 2018;3:17.

10. Song MS, Salmena L, Carracedo A, Egia A, Lo-Coco F, TeruyaFeldstein J, et al. The deubiquitinylation and localization of PTEN are regulated by a HAUSP-PML network. Nature. 2008;455:813-7.

11. van der Horst $A$, de Vries-Smits AM, Brenkman AB, van Triest $\mathrm{MH}$, van den Broek N, Colland F, et al. FOXO4 transcriptional activity is regulated by monoubiquitination and USP7/HAUSP. Nat Cell Biol. 2006;8:1064-73.

12. Morotti A, Panuzzo C, Crivellaro S, Pergolizzi B, Familiari U, Berger AH, et al. BCR-ABL disrupts PTEN nuclear-cytoplasmic shuttling through phosphorylation-dependent activation of HAUSP. Leukemia. 2014;28:1326-33.

13. D'Arcy $P$, Wang X, Linder S. Deubiquitinase inhibition as a cancer therapeutic strategy. Pharm Ther. 2015;147:32-54.

14. Kategaya L, Di Lello P, Rougé L, Pastor R, Clark KR, Drummond $\mathrm{J}$, et al. USP7 small-molecule inhibitors interfere with ubiquitin binding. Nature. 2017;550:534-8.

15. Turnbull AP, Ioannidis S, Krajewski WW, Pinto-Fernandez A, Heride C, Martin ACL, et al. Molecular basis of USP7 inhibition by selective small-molecule inhibitors. Nature. 2017;550:481-6.
16. Lamberto I, Liu X, Seo HS, Schauer NJ, Iacob RE, Hu W, et al. Structure-guided development of a potent and selective non-covalent active-site inhibitor of USP7. Cell Chem Biol. 2017;24:1490-500.

17. Pozhidaeva A, Valles G, Wang F, Wu J, Sterner DE, Nguyen P, et al. USP7-specific inhibitors target and modify the enzyme's active site via distinct chemical mechanisms. Cell Chem Biol. 2017;24:1501-12.

18. Desroses M, Altun M. The next step forward in ubiquitin-specific protease 7 selective inhibition. Cell Chem Biol. 2017;24:1429-31.

19. Gavory G, O’Dowd CR, Helm MD, Flasz J, Arkoudis E, Dossang A, et al. Discovery and characterization of highly potent and selective allosteric USP7 inhibitors. Nat Chem Biol. 2018; 14:118-25.

20. Carra G, Panuzzo C, Torti D, Parvis G, Crivellaro S, Familiari U, et al. Therapeutic Inhibition of USP7-PTEN network in chronic lymphocytic leukemia: a strategy to overcoming TP53 mutated/ deleted clones. Oncotarget. 2017;8:35508-22.

21. Chauhan D, Tian Z, Nicholson B, Kumar KG, Zhou B, Carrasco $\mathrm{R}$, et al. A small molecule inhibitor of ubiquitin-specific protease7 induces apoptosis in multiple myeloma cells and overcomes bortezomib resistance. Cancer Cell. 2012;22:345-58.

22. Fan YH, Cheng J, Vasudevan SA, Dou J, Zhang H, Patel RH, et al. USP7 inhibitor P22077 inhibits neuroblastoma growth via inducing p53-mediated apoptosis. Cell Death Dis. 2013;4:e867.

23. Adam K, Cartel M, Lambert M, David L, Yuan L, Besson A et al. A PIM-CHK1 signaling pathway regulates PLK1 phosphorylation and function during mitosis. J Cell Sci. 2018;10:131.

24. David L, Fernandez-Vidal A, Bertoli S, Grgurevic S, Lepage B, Deshaies D, et al. CHK1 as a therapeutic target to bypass chemoresistance in AML. Sci Signal. 2016;9:ra90.

25. Network TCGAR. Genomic and epigenomic landscapes of adult de novo acute myeloid leukemia. N Engl J Med. 2013;368: 2059-74.

26. Verhaak RG, Wouters BJ, Erpelinck CA, Abbas S, Beverloo HB, Lugthart S, et al. Prediction of molecular subtypes in acute myeloid leukemia based on gene expression profiling. Haematologica. 2009;94:131-4.

27. Hackl H, Steinleitner K, Lind K, Hofer S, Tosic N, Pavlovic S, et al. A gene expression profile associated with relapse of cytogenetically normal acute myeloid leukemia is enriched for leukemia stem cell genes. Leuk Lymphoma. 2015;56:1126-8.

28. Farge T, Saland E, de Toni F, Aroua N, Hosseini M, Perry R, et al. Chemotherapy-resistant human acute myeloid leukemia cells are not enriched for leukemic stem cells but require oxidative metabolism. Cancer Discov. 2017;7:716-35.

29. Eppert K, Takenaka K, Lechman ER, Waldron L, Nilsson B, van Galen $\mathrm{P}$, et al. Stem cell gene expression programs influence clinical outcome in human leukemia. Nat Med. 2011;17:1086-93.

30. Alonsa de Vega I, Martin Y, Smits VAJ. USP7 controls Chk1 protein stability by direct deubiquitination. Cell Cycle. 2014; 13:3921-6.

31. Zhang P, Wei Y, Wang L, Debeb BG, Yuan Y, Zhang J, et al. ATM-mediated stabilization of ZEB1 promotes DNA damage response and radioresistance through CHK1. Nat Cell Biol. 2014; 16:864-75.

32. An T, Gong Y, Li X, Kong L, Ma P, Gong L, et al. USP7 inhibitor P5091 inhibits wnt signaling and colorectal tumor growth. Biochem Pharmacol. 2017;131:29-39.

33. Petermann E, Maya-Mendoza A, Zachos G, Gillespie DA, Jackson DA, Caldecott KW. CHK1 requirement for high global rates of replication fork progression during normal vertebrate $\mathrm{S}$ phase. Mol Cell Biol. 2006;26:3319-26.

34. Lecona E, Rodriguez-Acebes S, Specks J, Lopez-Contreras AJ, Ruppen I, Murga M, et al. USP7 is a SUMO deubiquitinase essential for DNA replication. Nat Struct Mol Biol. 2016;23:270-7. 
35. Zhao GY, Lin ZW, Lu CL, Gu J, Yuan YF, Xu FK, et al. USP7 overexpression predicts poor prognosis in lung squamous cell carcinoma and large cell carcinoma. Tumor Biol. 2015;36:1721-9.

36. Ma M, Yu N. Ubiquitin-specific protease 7 expression is a prognostic factor in epithelial ovarian cancer and correlates with lymph node metastasis. Onco Targets Ther. 2016;9:1559-69.

37. Shan H, Li X, Xiao X, Dai Y, Huang J, Song J, et al. USP7 deubiquitinates and stabilizes NOTCH1 in T-cell acute lymphoblastic leukemia. Signal Transduct Target Ther. 2018;3:29.

38. Jin Q, Martinez CA, Arcipowski KM, Zhu Y, Gutierrez-Diaz BT, Wang KK, et al. USP7 cooperates with NOTCH1 to drive the oncogenic transcriptional program in $\mathrm{T}$ cell leukemia. Clin Cancer Res. 2018;25:222-39.
39. Lobry C, Oh P, Mansour MR, Look AT, Aifantis I. Notch signaling: switching an oncogene to a tumor suppressor. Blood. 2014;123:2451-9.

40. Wang S, Itoh M, Shiratori E, Ohtaka M, Tohda S. NOTCH activation promotes glycosyltransferase expression in human myeloid leukemia cells. Hematol Rep. 2018;10:7576.

41. Xu X, Zhao Y, Xu M, Dai Q, Meng W, Yang J, et al. Activation of notch signal pathway is associated with a poorer prognosis in acute myeloid leukemia. Med Oncol. 2011;28:483-9.

42. Takam Kamga P, Bassi G, Cassaro A, Midolo M, Di Trapani M, Gatti A, et al. Notch signaling drives bone marrow stromal cellmediated chemoresistance in acute myeloid leukemia. Oncotarget. 2016;7:21713-27. 